\title{
Measuring the corrugation amplitude of suspended and supported graphene
}

\author{
D. A. Kirilenko, ${ }^{1, *}$ A. T. Dideykin, ${ }^{2}$ and G. Van Tendeloo ${ }^{1}$ \\ ${ }^{1}$ EMAT, University of Antwerp, Groenenborgerlaan 171, B-2020 Antwerp, Belgium \\ ${ }^{2}$ Ioffe Institute, 194021, Politekhnicheskaya 26, St-Petersburg, Russia
}

(Received 11 November 2011; published 2 December 2011)

\begin{abstract}
Nanoscale corrugation is a fundamental property of graphene arising from its low-dimensional nature. It places a fundamental limit to the conductivity of graphene and influences its properties. However the degree of the influence of the corrugation has not been well established because of the little knowledge about its spectrum in suspended graphene. We present a transmission electron microscopy technique that enables us to measure the average corrugation height and length. We applied the technique also to measure the temperature dependence of the corrugation. The difference in corrugation between suspended and supported graphene has been illustrated.
\end{abstract}

DOI: 10.1103/PhysRevB.84.235417

PACS number(s): 63.22.Rc, 63.20.dd, 68.37.Lp, 68.65.Pq

\section{INTRODUCTION}

Graphene $^{1}$ as a materialized surface can be referred to as a "devil's invention," according to the well-known words of Wolfgang Pauli. Along with demonstrating outstanding ${ }^{2}$ and intriguing ${ }^{3}$ electronic properties that make graphene a very good prospective material for applications, it leaves plenty of riddles for fundamental research. The existence of graphene in stable form remains an astonishing phenomenon since a two-dimensional (2D) lattice was predicted to suffer from enormous undulations. ${ }^{4,5}$ The latter was highlighted when the roughness of suspended graphene was revealed. ${ }^{6}$ The roughness is shown to be an intrinsic property of graphene and is related to the corrugation usually observed by atomic force microscopy (AFM) or scanning tunneling microscopy (STM) in graphene deposited on a substrate. ${ }^{7,8}$ These techniques, however, cannot be applied on a nanoscale to suspended graphene, whereas supported graphene represents corrugation, which is severely affected by the interaction with the substrate. Moreover, supported graphene makes an intrinsic corrugation change under varying conditions impossible to notice. The corrugation decreases the conductivity of graphene $\mathrm{e}^{9,10}$ and influences its properties, but the degree of influence is still under debate because of a lack of experimental data on the corrugation amplitude of suspended graphene.

This work proposes a simple transmission electron microscopy (TEM) technique that enables us to measure the average corrugation amplitude and length and to estimate its overall spectrum. The technique is based on electron diffraction analysis. It is applicable to suspended graphene, and it allows us to carry out in situ studies on the corrugation change under varying conditions. We measured the temperature dependence of the corrugation spectrum and found an increase of the average wave vector with temperature, which is qualitatively consistent with theoretical studies. ${ }^{1-13}$ The measured average amplitude decreases with temperature, which seems rather unexpected and requires further investigation.

The technique can be applied to graphene supported by a relatively thin substrate. We found that suspended graphene sheets are indeed corrugated, but the amplitude is less than for graphene attached to a supporting film. This finding is in close connection with the observed difference in roughness parameters between suspended and supported graphene. ${ }^{14}$
The technique is based on electron-diffraction analysis. Electron diffraction is actually showing us a section through reciprocal space. Tilting the sample leaves us with various cross-sections, which can be combined to get an idea about corrugation spectrum. In the next section we will describe in more general terms the reciprocal space of a $2 \mathrm{D}$ corrugated crystal.

\section{RECIPROCAL SPACE OF A CORRUGATED 2D CRYSTAL}

For an ideally flat 2D crystal the density distribution in reciprocal space consists of rods perpendicular to the basal plane, but corrugation induces particular features. Figure 1(a) shows a schematic representation of the reciprocal space of corrugated graphene: considerably shortened rods, each surrounded by a diffuse cone related to the imperfectness.

Figure 1(b) shows an area around one rod in the reciprocal space of a corrugated crystal with designated sections obtained by electron diffraction. Different from the situation of a flat crystal, the rod, which appears as a bright spot of constant width, is rapidly fading out when tilting away from the basal plane $w=0$. There is a gradually broadening diffuse intensity around the rod, which is also the result of the rippling. The experiment [Fig. 2(c)] confirms the structure in the reciprocal space obtained by simulation.

In order to obtain quantitative information from the experimental data we have to analyze the reciprocal space of corrugated crystal in more detail. Let us consider a 2D crystal in the $X Y$ plane where the atoms have undergone out-of-plane shifts $h(x, y)$ along the $z$-axis. Then the density $F$ around a rod in reciprocal space $(u, v, w)$ of such a wavy crystal, neglecting the structural and atomic form factors, can be written as

$$
\begin{aligned}
F(u, v, w)= & \iint_{X Y} d y d x \exp (2 \pi i w h(x, y)) \\
& \times \exp (2 \pi i(u x+v y)) .
\end{aligned}
$$

Each cross-section $w=$ const is the Fourier transform of the phase-shifting field $\exp ((2 \pi \mathrm{iwh} x, y))$. In the case of a flat crystal, i.e., $h(x, y)=0$, expression (1) will be a $\delta$-function, thus, producing a uniform rod parallel to the $w$-axis. The presence of ripples causes the rod to fade out along the $w$-axis and diffuse intensity around the rod to appear: this follows 


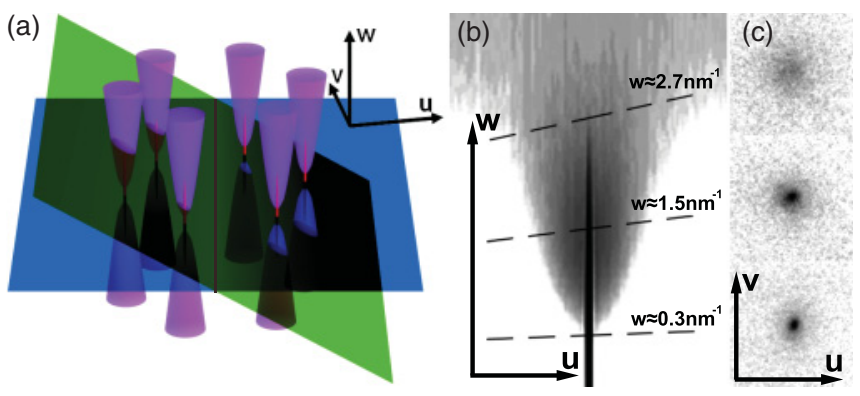

FIG. 1. (Color online) (a) Reciprocal space of rippled graphene intersected by the Ewald's sphere (idealized by the green/lighter gray plane). (b) Reciprocal space around one rod, and a scheme of various sections obtained from an electron-diffraction tilt series. (c) Experimental evolution of a single diffracted spot for increasing tilt angles, i.e., for increasing $w$-values.

from the expansion of the first exponential in (1) maintaining only the first-order term:

$$
F(u, v, w)=\delta(u, v) \times\left(1-2(\pi w)^{2}\left\langle h^{2}\right\rangle\right)+i(2 \pi w) h_{q}(u, v)
$$

where $\left\langle h^{2}\right\rangle$ is the average square of $h(x, y)$ or the crystal roughness and $h_{q}$ is the corrugation spectrum that is the Fourier transform of $h(x, y)$. The diffraction image is therefore a spot of intensity $I \propto\left(1-(2 \pi w)^{2}\left\langle h^{2}\right\rangle\right)$ situated in the center of the corrugation power spectrum image $\left|h_{q}\right|^{2}$. The image roughly shows the shape of the spectrum and the average wave vector but does not give any idea about its amplitude. The amplitude can be derived from the $I(w)$ change versus $w^{2}$, which depends on $\left\langle h^{2}\right\rangle$. Expression (2) is only valid if $(2 \pi w)^{2}\left\langle h^{2}\right\rangle$ is less than unity, so that $I(w)$ does not deviate too much from its maximum $I(0)$. If we assume that the ripples are not correlated, we can use the more general expression $I(w) \propto \exp \left(-(2 \pi w)^{2}\left\langle h^{2}\right\rangle\right),{ }^{15}$ which showed itself as a good approximation. Since the slope of the $\ln (I)$ versus $w^{2}$ equals $(2 \pi)^{2}\left\langle h^{2}\right\rangle$, it provides a measure for the corrugation average amplitude or crystal roughness.
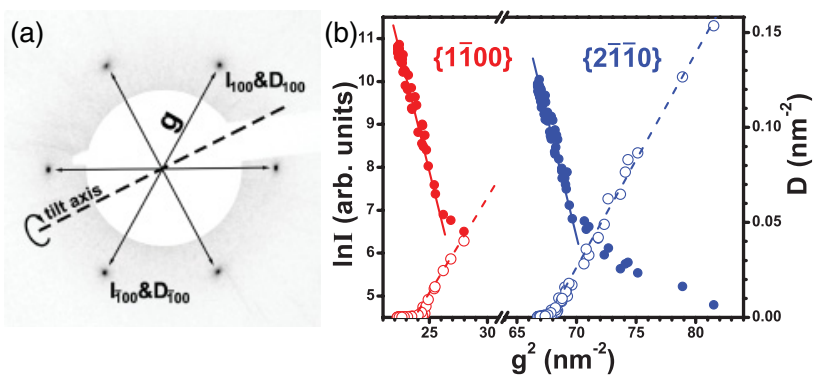

FIG. 2. (Color online) Graphene roughness measurement. (a) Diffraction pattern of graphene showing the measured values. (b) Plots of the spot intensity logarithm (solid circles) and dispersion (empty circles) versus the square of the distance between the spots. The red (light gray) symbols denote data for $\{1 \overline{1} 00\}$ spots and the blue (dark gray) symbols for $\{2 \overline{1} \overline{1} 0\}$ spots. The slopes of the curves are almost identical and correspond to the graphene roughness and the surface normal misorientation.
The characteristic corrugation length can be seen in diffraction at intermediate $w$ but can be determined more precisely from the diffuse ring broadening with increasing tilt since

$$
\begin{aligned}
D & =\iint_{\xi \zeta} d u d v\left(u^{2}+v^{2}\right)|F(u, v, w)|^{2} / \iint_{\xi \zeta} d u d v|F(u, v, w)|^{2} \\
& =\iint_{X Y} d x d y w^{2}\left(\left(\frac{\partial z}{\partial x}\right)^{2}+\left(\frac{\partial z}{\partial y}\right)^{2}\right) / \iint_{X Y} d x d y \\
& =w^{2}\left\langle|\nabla h|^{2}\right\rangle
\end{aligned}
$$

Thus, the slope of the spot dispersion $D$ versus $w^{2}$ equals the average square of the surface normal deviation $\left\langle|\nabla h|^{2}\right\rangle$. This is quite similar to an approach previously proposed. ${ }^{6}$ The average square of the corrugation wave vector $\left\langle|\vec{q}|^{2}\right\rangle=\left\langle|\nabla h|^{2}\right\rangle /\left\langle h^{2}\right\rangle$ is easily obtained from the measured values.

To simulate the waviness of graphene more accurately we could also take into account the in-plane shifts $\Delta x$ and $\Delta y$. But their magnitudes are expected to be considerably smaller. Moreover, they only cause a change of the spot intensity, which is independent on $w$; in other words, in-plane disorder does not affect the measurement of out-of-plane deviations.

In practice the calculated spot dispersion and measured intensity depend on choice of the origin $(u=0, v=0)$ in a spot image. Different choices correspond as if we define $h(x, y)$ relative to different coordinate systems. We used the center of mass of the spot as the origin that corresponds as if one defines the coordinate system so as to nullify average tilt of the crystal. Thus, the values of $\left\langle h^{2}\right\rangle$ and $\left\langle|\nabla h|^{2}\right\rangle$ were measured relative to the basic crystal plane.

The resolution of the technique is limited by the spatial coherency of the electron beam. The latter is about $70 \mathrm{~nm}$, which is of the order of the size of the aperture used. Convolution of point-spread function with (1) shows that the measured corrugation amplitude is an integral of the corrugation spectrum excluding waves longer than $70 \mathrm{~nm}$. Since the average corrugation length is measured to be about $10 \mathrm{~nm}$, the finite spatial coherency hides only an insignificant part of the spectrum, as is proved by our experimental data [Fig. 2(b)]. The main advantage of TEM, high electron-beam coherency, allows us to measure the corrugation amplitude directly from diffraction data, namely, from the diffraction spots intensities.

\section{EXPERIMENTAL RESULTS AND DISCUSSION}

As argued before, the roughness of graphene can be determined as a slope of the spot intensity logarithm $\ln (I)$ versus $w^{2}$. While $w$ itself cannot be directly measured on a diffraction pattern, one can notice that the square of the distance between two opposite spots $g^{2}$ equals $g^{2}=g_{0}{ }^{2}+w^{2}$, where $g_{0}$ is the reciprocal lattice basic vector. Thus, the slope angle of the $\ln (I)$ versus $g^{2}$ also equals $-(2 \pi)^{2}\left\langle h^{2}\right\rangle$, and the slope angle of the spot dispersion $D$ versus $g^{2}$ equals $\left\langle|\nabla h|^{2}\right\rangle$. This is valid since the electrons' wave vector in TEM is much larger than $g_{0}$, and the Ewald sphere can be idealized by a plane. Averaging of intensities and dispersions of opposite spots reduces the effect caused by Ewald-sphere curvature to the next order of vanishing. This approach eliminates the necessity to determine the actual graphene tilt angle or to adjust its orientation before the experiment. The only thing one needs 
for analysis is a clear diffraction pattern. Figure 2 represents a typical diffraction pattern of graphene and plots of the intensity logarithm and dispersion versus $g^{2}$ deduced from a tilt series. The tilt angle varied in the range from $-25^{\circ}$ to $+25^{\circ}$.

Points corresponding to all the spots of the $\{1 \overline{1} 00\}$ system are arranged along the same curves because of graphene's 6 -fold symmetry. The points related to the $\{2 \overline{1} \overline{1} 0\}$ system are arranged similarly, but they are shifted along the abscis and show a wider spread of $g^{2}$ values because the basic diffraction vector for the $\{2 \overline{1} \overline{1} 0\}$ spots is larger and the spots are further away from the tilt axis. The intensity logarithm follows a straight line until the bright spot dominates over the diffuse one, i.e., at tilt angles below ca. $10^{\circ}$. The dispersion reaches a linear dependence when only a diffuse spot remains and when the bright central contribution does not influence the measurements.

It is important to notice that the slope of dispersion at low $g^{2}$ values is almost negligible in comparison to its limit at high values and that the intensity logarithm has a linear dependence. This indicates that the ratio of the beam spatial coherency to the average corrugation length is very high and that pointspread-function finite width does not conceal a significant part of the spectrum.

The slope of the $\ln (I)$ lines are almost identical for the $\{1 \overline{1} 00\}$ and $\{2 \overline{1} \overline{1} 0\}$ spots and equal $1.2 \mathrm{~nm}^{2}$; this leads to a graphene rms roughness $\sqrt{\left\langle h^{2}\right\rangle}=1.7 \AA$. We also measured the average square of the surface inclination: $\left\langle|\nabla h|^{2}\right\rangle=0.011$, which means $6.0^{\circ}$. Finally, the average ripple wave vector is $0.67 \mathrm{~nm}^{-1}$, which corresponds to $10 \mathrm{~nm}$ in direct space and provides a measure of the corrugation lateral scale. The presented lateral scale does not mean a presence of any preferential length of corrugation or, in other words, any bump in the spectrum, but is similar to the term "the spatial extent" used in Ref. 6.

We applied the technique to graphene lying on an amorphous carbon film, thin enough to be transparent for electrons in TEM [Fig. 3(a)]. The film scatters some of the electrons and changes the spot intensities, but the effect decreases the intensities uniformly and therefore does not affect the slope of the $\ln (I)$ curve. We measured $\sqrt{\left\langle h^{2}\right\rangle}=2.7 \AA$ [Fig. 3(a)] for the
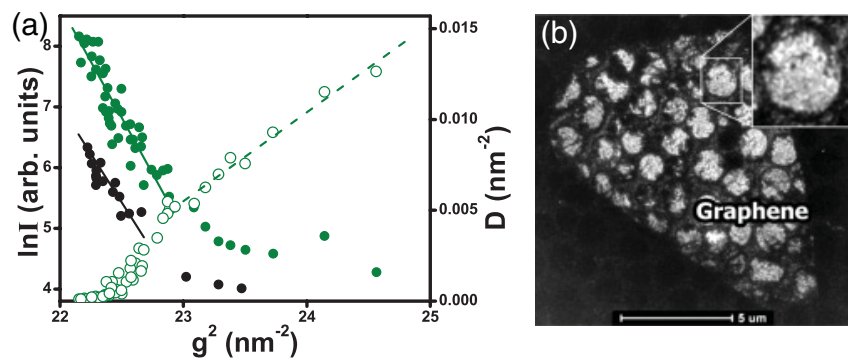

FIG. 3. (Color online) Supported graphene. (a) Plots of $\{100\}$ spot intensity logarithm (solid circles) and dispersion (open circles) of two flakes, stacked in a random orientation (green/light gray), and a flake supported by carbon film (black). (b) Graphene flake on a holey carbon film. Areas over the holes in the film are considerably brighter that show the difference between suspended and supported graphene. The $\{1 \overline{1} 00\}$ dark-field image was obtained using an ultrasmall objective aperture. One suspended area is shown enlarged in the inset. graphene supported by the film, which is considerably higher than for suspended graphene and is closer to values obtained by AFM and STM studies of supported graphene. ${ }^{7,8}$ Figure 3(b) is a dark-field TEM image obtained using an ultra-small objective aperture $\left(<10^{-4} \mathrm{rad}\right)$, so that the image can be considered as a map of the bright spot intensity. Although some contrast is partially caused by the film, the image can be considered as a good representation of the difference between suspended and supported graphene morphologies. The suspended area inhomogeneities might be caused by a curvature of the support and sagging of graphene; the latter is seen as a black rim at the edges of the holes [see inset of Fig. 3(b)]. Unfortunately, quantitative measurements of the average wave vector are considerably disturbed by the strong background of the carbon film. Nevertheless, they clearly illustrate a considerable decrease of corrugation lateral scale in comparison to suspended graphene, as was more precisely shown in Ref. 14.

Additionally, we investigated two graphene flakes stacked together at an arbitrary orientation, twisted by about $10^{\circ}$ with respect to each other. The measured roughness $\sqrt{\left\langle h^{2}\right\rangle}=2.8 \AA$ [Fig. 3(a)] is higher than for suspended graphene, whereas the average surface tilt $\left\langle|\nabla h|^{2}\right\rangle=0.004$ (or $3.9^{\circ}$ ) is considerably less. This implies a significant increase of the corrugation lateral scale up to $27 \mathrm{~nm}$. So, despite the higher roughness amplitude, the flakes stacked in a random orientation have a considerably smoother corrugation, which is closer to that of double-layer graphene, ${ }^{6,14}$ whereas the latter has much more deprived charge carriers mobility. So random stacking with another graphene can be used to reduce the negative effect of corrugation on conductivity.

We further determined the temperature dependence of the graphene corrugation. The most striking deviation from the intuitive expectation is the increase of the average corrugation height with decreasing temperature. This is deduced from the increased slope of the spot intensity at lower temperature (Fig. 4). However, the average surface tilt slightly decreases. This implies a significant decrease of the average wave vector and an increase of the corrugation length to $18 \mathrm{~nm}$ at $150 \mathrm{~K}$. The change of the average corrugation wave vector must lead to

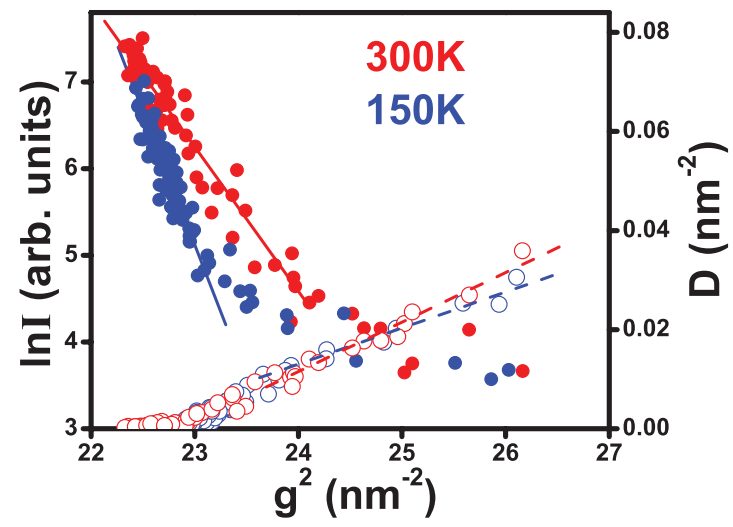

FIG. 4. (Color online) Spot intensity logarithm (solid) and dispersion (open) at various temperatures. The blue (dark gray) symbols correspond to $150 \mathrm{~K}$, whereas red (light gray) correspond to $300 \mathrm{~K}$. The increase of the corrugation amplitude with decreasing temperature is prominent. 
a huge alteration of the corrugation gain to the resistivity. ${ }^{9,10,16}$ This phenomenon may be partially responsible for the drastic behavior of the resistance of graphene with temperature, ${ }^{17,18}$ particularly for the difference between "dirty" and annealed graphene sheets.

The higher average amplitude at lower temperatures seems contradictory, since the flexural phonon spectrum should decrease at any wavelength together with the measured corrugation amplitude since it is the integral of the spectrum. We observed this effect independent of the direction of the temperature change. We also confirmed that 15 minutes of electron irradiation under our experimental conditions does not cause any noticeable change in the measured values. This is of course a consequence of the low current density we used for the diffraction experiments and confirms the intrinsic validity our data. The limited resolution of the technique causes some underestimation of the corrugation height, but the underestimation might only increase with an increase of the corrugation lateral scale. Therefore, the observed effect might only be underestimated because of the technique's limited resolution. It is also important to notice that separate measurements over one hole in the carbon film gave approximately the same values. The measured values of the average corrugation amplitude and length are therefore unique characteristics of the suspended area. However there is some spread in data obtained for different holes; this is most probably related to the dependence of the corrugation on the size of the suspended area, which varied in range $0.5-5 \mu \mathrm{m}$. It is also important that the aforementioned temperature effect is observed independently on a hole size, despite the difference in absolute values of measured parameters. Graphene used in our experiments was obtained by an exfoliation method described in Ref. 19; this material provides a sharp diffraction pattern proving an undisturbed crystalline structure. Some impurities were detected in the material, but the strong temperature dependence of the morphology suggests that the impurities have not significantly suppressed the elastic properties of the graphene.

The measured corrugation height is an average over a limited area of about $100 \mathrm{~nm}$, thus, its decrease with increasing temperature means a decrease of the corrugation spectrum at wave lengths below $100 \mathrm{~nm}$. Apparently, long wave-length undulations are involved in the decrease, because an increase of the average wave vector, roughness exponent, ${ }^{14}$ and average interatomic distance ${ }^{20}$ means an increase of the short-length corrugations.

The measured values might differ somewhat from the actual characteristics, defined as average across the full corrugation spectrum, because of the possible divergence of the spectrum around zero. ${ }^{21,22}$ Nevertheless, it leaves our qualitative conclusions unaltered. Moreover, it would imply a characteristic length comparable to the size of the measured area. The plot of the dispersion [Fig. 2(b)] will not drastically change its slope at intermediate tilts. We would like to emphasize that theoretical models of graphene corrugation presented in literature (e.g., Refs. 11,12, 22, and 23) show quite different spectrum dependences at small wave vectors. This withheld us from choosing any particular model in frames of our work, but the experimental results obtained suggest that corrugation spectrum is not likely to be strongly divergent around zero point. Additionally, an increase of the long corrugations might also cause an additional growth of the corrugation lateral scale, so that the temperature dependence of the characteristic wave vector is stronger than expected from literature. ${ }^{1-13}$ The anomalous behavior could eventually be connected with the transition presented in Ref. 23, but this is unlikely since no significant strain appears in our experiments. The anomalous temperature dependencies of lattice properties ${ }^{24}$ are responsible to some degree for the effect; however, they cannot fully explain its magnitude. We suppose that the observed effect might be connected to the interaction between structural and charge fluctuations, because this will significantly affect the temperature dependence, as was shown in Ref. 12.

\section{CONCLUSIONS}

We presented a technique for measuring the average height and the lateral size of nanoscale corrugation in suspended graphene. These are crucial parameters of the corrugation spectrum and results are important for low-dimensional structure physics. The technique is applicable no matter whether the corrugation is dynamic or static. The results prove the essential role of anharmonicity in the corrugation behavior. The roughness of suspended graphene is less than that of graphene stacked on a substrate. We also found that two graphene flakes stacked together at random orientation are less corrugated. The observed decrease of the membrane roughness with temperature requires further theoretical investigations. Our experimental data suggest that the drastic increase of resistivity with temperature is correlated with the nanoscale corrugation behavior of suspended graphene.

\section{ACKNOWLEDGMENTS}

G.V.T. acknowledges the ERC Grant No. 246791COUNTATOMS. A.T.D. acknowledges support of the Ministry of education and science of Russian Federation (Contract No. 14.740.11.0593).
*Corresponding author: zumsisai@gmail.com

${ }^{1}$ K. S. Novoselov, A. K. Geim, S. V. Morozov, D. Jiang, Y. Zhang, S. V. Dubonos, I. V. Grigorieva, and A. A. Firsov, Science 306, 666 (2004).

${ }^{2}$ K. S. Novoselov, A. K. Geim, S. V. Morozov, D. Jiang, M. I. Katsnelson, I. V. Grigorieva, S. V. Dubonos, and A. A. Firsov, Nature 438, 197 (2005).

\footnotetext{
${ }^{3}$ A. K. Geim and K. S. Novoselov, Nat. Mater. 6, 183 (2007).

${ }^{4}$ L. D. Landau and E. M. Lifshitz, Statistical Physics Part I, (Pergamon, Oxford, 1980), p. 207.

${ }^{5}$ N. D. Mermin, Phys. Rev. 176, 250 (1968).

${ }^{6}$ J. C. Meyer, A. K. Geim, M. I. Katsnelson, K. S. Novoselov, T. J. Booth, and S. Roth, Nature 446, 60 (2007).
} 
${ }^{7}$ V. Geringer, M. Liebmann, T. Echtermeyer, S. Runte, M. Schmidt, R. Rückamp, M. C. Lemme, and M. Morgenstern, Phys. Rev. Lett. 102, 076102 (2009).

${ }^{8}$ M. Ishigami, J. H. Chen, W. G. Cullen, M. S. Fuhrer, and E. D. Williams, Nano Lett. 7, 1643 (2007).

${ }^{9}$ M. I. Katsnelson and A. K. Geim, Philos. Trans. R. Soc. A 366, 195 (2008).

${ }^{10}$ E. V. Castro, H. Ochoa, M. I. Katsnelson, R. V. Gorbachev, D. C. Elias, K. S. Novoselov, A. K. Geim, and F. Guinea, Phys. Rev. Lett. 105, 266601 (2010).

${ }^{11}$ A. Fasolino, J. H. Los, and M. I. Katsnelson, Nat. Mater. 6, 858 (2007).

${ }^{12}$ D. Gazit, Phys. Rev. B 80, 161406(R) (2009).

${ }^{13}$ F. L. Braghin and N. Hasselmann, Rhys. Rev. B 82, 035407 (2010).

${ }^{14}$ A. Locatelli, K. R. Knox, D. Cvetko, T. O. Mentes, M. A. Nino, S. Wang, M. B. Yilmaz, P. Kim, R. M. Osgood, and A. Morgante, ACS Nano 4, 4879 (2010).

${ }^{15}$ H.-N. Yang, T.-M. Lu, and G.-C. Wang, Phys. Rev. B 47, 3911 (1993).
${ }^{16}$ E.-A. Kim and A. H. Castro Neto, Europhys. Lett. 84, 57007 (2008).

${ }^{17}$ S. V. Morozov, K. S. Novoselov, M. I. Katsnelson, F. Schedin, D. C. Elias, J. A. Jaszczak, and A. K. Geim, Phys. Rev. Lett. 100, 016602 (2008).

${ }^{18}$ K. I. Bolotin, K. J. Sikes, J. Hone, H. L. Stormer, and P. Kim, Phys. Rev. Lett. 101, 096802 (2008).

${ }^{19}$ A. T. Dideykin, A. E. Aleksenskiy, D. Kirilenko, P. Brunkov, V. Goncharov, M. Baidakova, D. Sakseev, and A. Ya. Vul', Diam. Relat. Mater. 20, 105 (2011).

${ }^{20}$ M. Pozzo, D. Alfe, P. Lacovig, P. Hofmann, S. Lizzit, and A. Baraldi, Phys. Rev. Lett. 106, 135501 (2011).

${ }^{21}$ D. R. Nelson and L. Peliti, J. Physique 48, 1085 (1987).

${ }^{22}$ J. H. Los, M. I. Katsnelson, O. V. Yazyev, K. V. Zakharchenko, and A. Fasolino, Phys. Rev. B 80, 121405R (2009).

${ }^{23}$ N. Abedpour, R. Asgari, and M. R. Tabar, Phys. Rev. Lett. 104, 196804 (2010).

${ }^{24}$ K. V. Zakharchenko, M. I. Katsnelson, and A. Fasolino, Phys. Rev. Lett. 102, 046808 (2009). 